\title{
Reología, comportamiento físico-mecánicos y de durabilidad de hormigones con nanosílice
}

\author{
E. Téllez Girón ${ }^{1}$, A .N. Martín Acosta ${ }^{1}$, A. Pérez Hernández ${ }^{1}$, U. Verdecia Rodríguez ${ }^{2}$ \\ ${ }^{1}$ Centro Técnico para el Desarrollo de los Materiales de Construcción, Carretera Casa Blanca y Calle 70. Reparto Bahía. \\ Municipio Regla. CP 11200. Ciudad de la Habana, Cuba. \\ ${ }^{2}$ Empresa Nacional de Investigaciones Aplicadas. Ministerio de la Construcción. Carretera Central Km. 4 1⁄2 vía a Santiago. \\ Bayamo, Granma. Cuba.
}

\section{Información del artículo \\ DOI: \\ http://dx.doi.org/10.21041/ra.v2 $\underline{i 2.29}$ \\ Artículo recibido el 02 de Marzo de 2012, revisado bajo \\ las políticas de publicación de la Revista ALCONPAT y aceptado el 17 de Mayo de 2012. Cualquier discusión, incluyendo la réplica de los autores se publicará en el primer número del año 2013 siempre y cuando la información se reciba antes del cierre del tercer número del año 2012}

\section{(C) 2012 ALCONPAT Internacional}

Información Legal

Revista ALCONPAT, Año 2, No. 2, Mayo Agosto 2012, es una publicación cuatrimestral de la Asociación Latinoamericana de Control de Calidad, Patología y Recuperación de la Construcción, Internacional, A.C., Km. 6 , antigua carretera a Progreso, Mérida Yucatán, C.P. 97310 , Tel.5219997385893, alconpat.int@gmail.com, Página Web: www.alconpat.org

Editor responsable: Dr. Pedro Castro Borges. Reserva de derechos al uso exclusivo No.042013-011717330300-203, eISSN 2007-6835, ambos otorgados por el Instituto Nacional de Derecho de Autor. Responsable de la última actualización de este número, Unidad de Informática ALCONPAT, Ing. Elizabeth Sabido Maldonado, Km. 6, antigua carretera a Progreso, Mérida Yucatán, C.P. 97310, fecha de publicación: 30 de Mayo de 2012

Las opiniones expresadas por los autores no necesariamente reflejan la postura del editor. Queda totalmente prohibida la reproducción total o parcial de los contenidos e imágenes de la publicación sin previa autorización de la ALCONPAT Internacional A.C.

\section{RESUMEN}

La nanosílice es una de las aplicaciones de la nanotecnologia en la construcción, a base de sílica amorfa nanométrica, que se emplea igual que la microsílice, pero con la ventaja que no produce contaminación al medio ambiente y la agresión a la salud por inhalación de ésta.

Se dan los resultados de la caracterización física de dos compuestos de nanosílice comerciales mediante los métodos de la caracterización de aditivos y el empleo de la microscopia para la determinación de compuestos fundamentales, de sus actividades puzolánicas en morteros, así como la determinación de la absorción capilar, velocidad ultrasónica, masa volumétrica, coeficientes de resistencia a la penetración de agua, coeficiente de absorción de agua y porosidad efectiva en hormigones elaborados con los compuestos evaluados y en hormigones con aditivos superplastificantes y tobas zeolitizadas como patrones de comparación. Los valores demuestran un mejor comportamiento de durabilidad en los hormigones elaborados con nanosílice.

Palabras Clave: nanotecnología; nanosílice; microsílice; aditivos químicos; adiciones.

\section{ABSTRACT}

Nanosilice based in nanometric amorphous silica is one of the aplications of nanotechnology in the building industry, that has the same use than microsilica, but with the advantage that it does not produce environmental pollution or respiratory complains due to inhalation.

We give the results of the physical characterization of two commercial nanosilice compounds by the admixtures characterization methods and electronic microscopy analysis to determine the fundamental compound, its pozzolanic activity in mortars, as well as its capillary absorption, ultrasonic pulse speed, volumetric mass, water penetration resistance coefficient, water absorption coefficient and effective porosity in concrete with nanosilice and in ordinary concrete with natural pozzolans and superplasticizer admixtures like comparative patterns. Results show a better behavior of durability in concretes with nanosilice.

Keywords: nanotechnology; nanosilica; microsilica; chemical admixtures; additions.

Autor de contacto: Elena Téllez Girón (elena@ctdmc.co.cu) 
Revista ALCONPAT, Volumen 2, Número 2, Mayo - Agosto 2012, Páginas 82 - 88

\section{INTRODUCCIÓN}

En estos momentos la nano- tecnología no es solo los nano-componentes o en aparatos ultra pequeños que están siendo desarrollados por científicos para la lucha contra enfermedades malignas, la biotecnología, la física y otras ramas, también en el sector de la construcción empieza a entrar en el mundo de los avances tecnológicos y se está empezando a utilizar la nanotecnología, aportando mejoras significativas en la construcción de edificaciones, autopistas y puentes mediante la aplicación de materiales y maquinarias producidos a partir del reordenamiento de átomos y moléculas, con nuevas propiedades únicas y bajo costo. Este es el caso del desarrollo de nuevos materiales a base de sílica amorfa nanométrica. Las nanopartículas de sílice generalmente mejoran las propiedades reológicas y mecánicas de los cementos y hormigones, pues como se sabe por la física y la química, las partículas de mil veces más pequeños diámetro tienen mayor reactividad al tener mayor superficie de contacto, lo que incrementa su efectividad, estabilidad y propiedades. La investigación desarrollo entorno a los nano-materiales y en especial la nanosílice en América Latina ha aportado una moderada experiencia y aplicaciones por ser productos de última generación en el mercado internacional. Cuba, en la medida que se siga revitalizando la economía, se seguirán diseñando y ejecutando obras para el sector inmobiliario y turístico en las cuales cada vez son mayores los requisitos de esbeltez estructural con altas cuantías de acero de refuerzo, la colocación del hormigón se puede convertir en un serio problema si no se asume la utilización del hormigones con alta resistencia y alto grado de durabilidad. En este sentido es muy positivo prepararse y avanzar en el estudio de este importante material que parece se impondrá en un futuro inmediato.

\section{PROCEDIMIENTO EXPERIMENTAL}

En el trabajo se realizó la caracterización física y química de los compuestos de nanosílice, pruebas en pasta de cemento con el objetivo de analizar el efecto que ejercen dos nanopartículas de sílice comerciales en la matriz de cemento Pórtland, su efecto como adición en morteros y las modificaciones de las propiedades de los hormigones con nanosílice comparados con hormigones elaborados con aditivos químicos y adición de puzolana natural con actividad puzolánica.

En caracterización física se emplearon los ensayos correspondientes a las Normas NC 271-2003 Parte 1-7 "Aditivos químicos métodos para su evaluación y la determinación de la composición química", se utilizó el microscopio electrónico de barrido, solo cualitativamente pues el producto final para llevarla sequedad se obtuvo un plástico gomoso, que no permitió otras determinaciones. En los ensayos en pasta de cemento se determinaron la influencia de estos aditivos en la plasticidad, reducción de agua y permanencia de la acción con el tiempo (pruebas reológicas), así como la determinación de las dosis óptimas del producto, mediante el procedimiento del tiempo de caída mediante el cono Marsh y las pruebas de expansión por fluidez por el método del minicono (mini-slump) simultáneamente. Las mezclas se prepararon con la relación a/c en 0,4 y se añadió el aditivo en las proporciones de 0-0,6-1,2-1,8-2,4 y 3,0 \% respecto al peso del cemento del aditivo Compuesto 1 y de 0-0,5-1,0-1,5 y 2,0 \% del aditivo Compuesto 2 .

El índice de actividad puzolánica de los compuestos de nanosílice se determinó por la variación de las resistencias a flexión y compresión a 7 y 28 días de los morteros elaborados con estos compuestos y sin ellos, según se establece en la norma ASTM C 618 el índice de actividad puzolánica. Los morteros se elaboraron con las proporciones de 1,8\% del compuesto C-1 y $1,5 \%$ del peso del cemento e igual fluidez que el mortero sin aditivo.

Para los ensayos en hormigones se elaboraron seis series con una dosificación calculada para una marca de $30 \mathrm{MPa}$ a los 28 días, y una relación a/c igual a 0,4 con los siguientes compuestos: 
Revista ALCONPAT, Volumen 2, Número 2, Mayo - Agosto 2012, Páginas 82 - 88

a. Con aditivo superplastficate a base de naftaleno sulfonado recomendado para la tecnología de prefabricado sin adición de puzolana natural, identificado como $(\mathrm{N})$

b. Adición del compuesto C-1 de nanosílice recomendado para el prefabricado $(\mathrm{G})$

c. Con aditivo superplastificante a base de naftaleno sulfonado recomendado par la tecnología del prefabricado y puzolana natural (NP)

d. Con el aditivo superplastificante a base de naftaleno sulfonado modificado recomendado para el premezclado $(\mathrm{RC})$

e. Adición del compuesto C-2 de nanosílice recomendado para el premezclado (M)

f. Con el aditivo superplastificante a base de naftaleno modificado recomendado para el hormigón premezclado y puzolana natural (RP)

\subsection{Ensayos al hormigón:}

Velocidad ultrasónica: Para el ensayo se utilizó el equipo Proceq TICO, que mide de 15 a 6550 milisegundos. Se aplicó el procedimiento establecido por la norma NC 231: 2002 "Determinación, interpretación y aplicación de la velocidad del pulso ultrasónico en el hormigón". Las mediciones en tres puntos de cada probeta. A las que previamente se le había determinado con exactitud el diámetro y la longitud (distancia a recorrer). La Red Durar establece que este ensayo tiene por objetivo verificar la homogeneidad (uniformidad y calidad relativa) del hormigón y detectar fallas internas (presencia de vacíos) introducidas durante fabricación, fisuras y otras imperfecciones, así como monitorear las variaciones debido a la agresividad del medio, con el tiempo.

Masa volumétrica. Se determinó según el procedimiento habitual de medición del peso y las dimensiones.

Absorción de agua por permeabilidad. Este ensayo fue realizado por lo establecido con la norma JIS 1404:1977 "Método de ensayo para adiciones impermeables del cemento en hormigones y morteros". Para el ensayo se empleó el permeabilímetro Alemán firma Toni-Technik con una junta de goma de diámetro $\varnothing 50 \mathrm{~mm}$ de abertura.

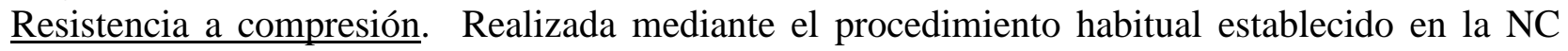
244:2003

Porosidad Efectiva, Resistencia a la penetración del agua y Absorción capilar. Determinación de éstos coeficientes se determinaron según lo establecido en la norma cubana NC 345: 2005 Hormigón endurecido. Determinación de la absorción de agua por capilaridad que utiliza el método propuesto por Goran Fagerlund.

\subsection{Materiales empleados}

Nanosílice. Para el trabajo se suministraron dos muestras de nanosílice, las cuales se recibieron en recipientes plásticos de $25 \mathrm{Kg}$, identificadas como Compuesto 1 (C-1) y Compuesto 2 (C-2) que según el productor, el primero es un aditivo para hormigón de la línea ecológica, a base de sílice en estado líquido con partículas de tamaño nanométrico del orden de 3 a 150 nanómetros que sustituye a la combinación aditivo-microsílice en todas sus aplicaciones y el segundo además de superplastificante - autocompactante, en estado líquido, gran reductor de agua, de alta actividad basado en nanomateriales de última generación. En la Tabla 1 se muestran las características físicas de los compuestos 1 y 2 comparadas con la microsílice

Cemento. Del tipo Pórtland de marca P-350 kg/cm² procedente de la fábrica René Arcay, Mariel, Provincia La Habana.

Áridos. Árido fracción 0-4,76 mm del yacimiento "Arimao", fracción 4,76-9,52 mm del yacimiento "Alacranes" ambos ubicados en la provincia de Matanzas, 9,52-19,1 mm del yacimiento "Dragón Camoa", La Habana. 
Revista ALCONPAT, Volumen 2, Número 2, Mayo - Agosto 2012, Páginas 82 - 88

Puzolana natural. Es una puzolana natural con propiedad puzolánica clasificadas como del tipo Clinoptilolita - Heulandita, del yacimiento de Tasajera de Villa Clara. Para el trabajo se tomó una muestra de la fracción menor de $0,8 \mathrm{~m}$

Aditivos superplastificantes. A los efectos de comparar los resultados del efecto de las nanosílice, se realizaron ensayos en hormigones con los aditivos superplastificantes para el premezclado y para el prefabricado conjuntamente con la adición de la puzolana natural que es lo que se emplea actualmente en el país.

\section{RESULTADOS}

Tabla 1. Características de los compuestos 1 y 2 comparadas con la microsílice

\begin{tabular}{|c|c|c|c|c|}
\hline Producto & $\begin{array}{c}\text { Densidad } \\
(\mathbf{g} / \mathbf{l})\end{array}$ & $\begin{array}{c}\text { Tamaño de partícula } \\
(\mathbf{n m})\end{array}$ & $\begin{array}{c}\text { Área Específica de } \\
\text { superficie }\left(\mathbf{m}^{\mathbf{2}} \mathbf{g}\right)\end{array}$ & Apariencia \\
\hline Nanosílice & 1,5 & $3-150$ & $20-1000$ & Líquido \\
\hline Microsílice & 0,3 & $200-1000$ & $15-20$ & Polvo \\
\hline
\end{tabular}

Tabla 2. Propiedades físicas de las muestras de nanosílice

\begin{tabular}{|c|c|c|c|c|}
\hline Propiedad & $(C-1)$ & $\begin{array}{c}\text { Valor de la } \\
\text { Ficha }\end{array}$ & $(C-2)$ & $\begin{array}{l}\text { Valor de } \\
\text { la Ficha }\end{array}$ \\
\hline \multirow{9}{*}{$\begin{array}{c}\text { Estado físico } \\
\text { Color } \\
\text { sólido } \\
\text { Densidad } \\
\text { pH } \\
\text { Viscosidad Ford } \Phi=2 \\
\text { Tensión superficial } \\
\text { Contenido de Cloruros } \\
\text { Solubilidad }\end{array}$} & L. T & LV & L. V & L. V \\
\hline & C. C. & C. C & C.O & C. $\mathrm{O}$. \\
\hline & $15,85 \%$ & - & 28,92 & - \\
\hline & $1,02\left(\mathrm{~g} / \mathrm{cm}^{2}\right)$ & $1,01-1,05$ & 1,05 & $1,10-1,14$ \\
\hline & 5,65 & $5 \pm 1$ & 5,77 & $6 \pm 1$ \\
\hline & $62 \mathrm{seg}$. & 12-14seg. & 77 & $24 \pm 2 \mathrm{seg}$ \\
\hline & $36,6(\mathrm{mN} / \mathrm{m})$ & - & 35,7 & - \\
\hline & 0,048 & $<0,1 \%$ & 0,044 & $<0,1 \%$ \\
\hline & $99,58(\%)$ & - & 99,55 & - \\
\hline
\end{tabular}

C.C : Marron Claro, C.O : Marron Oscuro, L.T: Líquido Turbio, V: Líquido Viscoso

Tabla 3. Composición química cualitativa

\begin{tabular}{|c|c|c|c|c|c|c|c|c|c|c|c|c|}
\hline \multirow[t]{2}{*}{ No. } & \multicolumn{6}{|c|}{ Compuesto 1} & \multicolumn{6}{|c|}{ Compuesto 2} \\
\hline & $\mathbf{C}$ & $\mathbf{O}$ & $\mathbf{N a}$ & $\overline{A u}$ & $\mathbf{S}$ & $\mathbf{S i}$ & $\mathrm{C}$ & $\mathbf{O}$ & $\mathbf{N a}$ & $\overline{\mathrm{Au}}$ & $\mathbf{S}$ & $\mathbf{S i}$ \\
\hline 1 & $\mathrm{PM}$ & $\mathrm{P}$ & $\mathrm{PP}$ & PP & PP & $\mathrm{P}$ & $\overline{\mathrm{PM}}$ & $\mathrm{P}$ & PP & $\mathrm{PP}$ & $\mathrm{PP}$ & $\mathrm{P}$ \\
\hline 2 & PM & $\mathrm{P}$ & PP & PP & PP & $\mathrm{P}$ & PM & $\mathrm{P}$ & PP & PP & PP & $P$ \\
\hline 3 & $\mathrm{PM}$ & $\mathrm{P}$ & PP & PP & PP & $\mathrm{P}$ & PM & $\mathrm{P}$ & $\mathrm{PP}$ & PP & PP & $\mathrm{P}$ \\
\hline 4 & PM & $\mathrm{P}$ & PP & PP & PP & $\mathrm{P}$ & PM & $\mathrm{P}$ & PP & PP & PP & $\mathrm{P}$ \\
\hline 5 & PM & $\mathrm{P}$ & PP & PP & PP & $\mathrm{P}$ & PM & $\mathrm{P}$ & PP & PP & PP & $\mathrm{P}$ \\
\hline
\end{tabular}

PM: Presencia mayoritaria P: Presencia PP: Poca presencia

\subsection{Caracterización física}

En la Tabla 2 se observa en ambos aditivos que aunque está dentro de lo especificado el pH resulta ser bajo, lo que no es del todo conveniente pues promueve la corrosión, esto resulta menos preocupante que en el caso de otros aditivos, dada la baja proporción en la que se dosifica. Tanto la densidad, el contenido de sólido como la viscosidad son bajas. 
Revista ALCONPAT, Volumen 2, Número 2, Mayo - Agosto 2012, Páginas 82 - 88

\subsection{Caracterización química}

En la Tabla 3 se puede observar que los elementos fundamentales presentes acorde a los espectros son el carbono, el oxígeno y la sílice, lo cual resulta lógico por ser compuestos orgánicos con adición de sílice amorfa. Otros elementos se encuentran en forma de trazas. La sílice presente en ambas muestran se encuentran enlazadas de forma amorfa a las cadenas carbonadas.

Tabla 4. Resultados de los ensayos en pasta de cemento

\begin{tabular}{|c|c|c|c|c|}
\hline Nanosílice & $\begin{array}{c}\text { \% respecto al } \\
\text { peso del } \\
\text { cemento }\end{array}$ & $\begin{array}{c}\text { \% } \\
\text { Incremento } \\
\text { del área }\end{array}$ & $\begin{array}{c}\text { Mantenimiento de } \\
\text { la plasticidad } \\
\text { (minutos) }\end{array}$ & $\begin{array}{c}\text { \% de } \\
\text { reducción } \\
\text { de agua }\end{array}$ \\
\hline Compuesto 1 (C-1) & 0,6 & 4,5 & 180 & 26 \\
\hline & 1,2 & 5,6 & 190 & 28 \\
\hline & 1,8 & 5,9 & 240 & 33 \\
\hline & 2,4 & 6,6 & 200 & 35 \\
\hline & 3,0 & 7,0 & 190 & 36 \\
\hline Compuesto 2(C-2) & 0,5 & 5,2 & 185 & 30 \\
\hline & 1,0 & 6,2 & 198 & 32 \\
\hline & 1,5 & 6,9 & 250 & 35 \\
\hline & 2,0 & 6,8 & 210 & 38 \\
\hline
\end{tabular}

En la Tabla 4 se observa que tanto el compuesto 1 (C-1) como el 2 (C-2) lograron un incremento notable de la capacidad plastificante, resultando mayor a medida que se incrementa el \% adicionado. También se obtuvo un tiempo de permanencia de la acción superior a 3 horas y la reducción de agua para el valor medio de la dosis fue de mas del $30 \%$ en ambos casos por lo que ambas sustancias pueden ser comparadas con aditivos reductores de agua-superplastificante.

El \% óptimo mediante el minicono y el cono Marsh se obtuvo en 1,8 y 1,5\% para el compuesto 1 y para el compuesto 2 , respectivamente.

Tabla 5. Propiedades físicas y físico-mecánicas de los morteros

\begin{tabular}{|l|c|c|c|}
\hline \multirow{2}{*}{ Compuesto empleado } & \multirow{2}{*}{ Edad } & $\begin{array}{c}\text { Resistencia Flexión } \\
\text { ( MPa) }\end{array}$ & $\begin{array}{c}\text { Resistencia } \\
\text { Compresión (MPa) }\end{array}$ \\
\hline Patrón & \multirow{3}{*}{7 días } & 7,6 & 34 \\
\hline Compuesto 1 (C-1) & \multirow{2}{*}{28 días } & 8,8 & 48,9 \\
\cline { 4 - 4 } & & 8,9 & 51,9 \\
\hline Compuesto 2 (C-2) & \multirow{2}{*}{ Patrón } & 12,1 & 45,3 \\
\hline Compuesto 1 (C-1) & 13,2 & 52,7 \\
\hline Compuesto 2 (C-2) & & 13,8 & 56,2 \\
\hline
\end{tabular}

Tabla 6. Resultados del cumplimiento de requisitos físicos de idoneidad como mineral activo

\begin{tabular}{|l|c|c|c|}
\hline \multicolumn{2}{|c|}{ Requerimientos físicos (ASTM C 618) } & Compuesto 1 & Compuesto 2 \\
\hline Finura \%. Retenido tamiz 45 $\mathrm{m}$ ( \% máx.) & $\mathbf{3 4}$ & $\begin{array}{c}\text { Líquido con } \\
\text { partículas } \\
\text { nanométricas }\end{array}$ & $\begin{array}{c}\text { Líquido con partículas } \\
\text { nanométricas }\end{array}$ \\
\hline Índice de Actividad. Cemento Pórtland mínimo \\
\hline Edad 7 días. \% mín. del mortero s/ adición & $\mathbf{7 5}$ & $115 \%$ & $1435 \%$ \\
\hline Edad 28 días \% mín. del mortero s/adición & $\mathbf{7 5}$ & $116 \%$ & $124,0 \%$ \\
\hline
\end{tabular}

Reología, comportamiento físico-mecánicos y de durabilidad de hormigones con nanosílice 86 
Revista ALCONPAT, Volumen 2, Número 2, Mayo - Agosto 2012, Páginas 82 - 88

Las resistencias tanto a 7 como a 28 días (Tabla 5) fueron muy superiores que las del mortero sin aditivo aproximadamente de 1,2\% mayor y el Índice de Actividad evaluado por la norma ASTM 618 establece que un compuesto tiene esta actividad cuando su resistencia es al menos un $75 \%$ superior al mortero patrón. En la Tabla 6 se observa que en el caso del compuesto C-1 es de 115 y 116 y C-2 es de 143 y 124 lo que corrobora que poseen actividad puzolánica.

Tabla 7. Materiales, propiedades y resultados de los hormigones en las variantes estudiadas

\begin{tabular}{|c|c|c|c|c|c|c|c|}
\hline \multirow{2}{*}{\multicolumn{2}{|c|}{ Materiales }} & \multirow{2}{*}{\multicolumn{2}{|c|}{$\frac{\mathbf{N}}{1 \mathrm{~m}^{3}}$}} & $\mathbf{G}$ & \multirow{2}{*}{\multicolumn{2}{|c|}{$\frac{\mathbf{R C}}{1 \mathrm{~m}^{3}}$}} & $\mathbf{M}$ \\
\hline & & & & $1 \mathrm{~m}^{3}$ & & & $1 \mathrm{~m}^{3}$ \\
\hline Cemento Pórtland Normal & $\mathrm{kg}$ & 400 & 400 & \multirow{11}{*}{$\begin{array}{c}400 \\
987 \\
463 \\
564 \\
- \\
1,8 \\
160 \\
144 \\
0,396 \\
210 \\
30 \\
\end{array}$} & 400 & 400 & \multirow{4}{*}{$\begin{array}{l}400 \\
987 \\
463 \\
564\end{array}$} \\
\hline Árido Fino Arimao & $\mathrm{kg}$ & 987 & 987 & & 987 & 987 & \\
\hline Arido Medio Alacranes & $\mathrm{kg}$ & 463 & 463 & & 463 & 463 & \\
\hline Gravilla Dragón Camoa & $\mathrm{kg}$ & 564 & 564 & & 564 & 564 & \\
\hline Puzolana, Tasajera & $\mathrm{Kg}$ & - & 40 & & - & 40 & - \\
\hline Aditivo real , & $\%$ & 1,6 & 1,9 & & 1,4 & 1,8 & 1,5 \\
\hline Agua, litros & & 160 & 160 & & 160 & 160 & 160 \\
\hline Agua real, litros & & 160 & 168 & & 176 & 176 & 128 \\
\hline $\mathrm{a} / \mathrm{c}$ real & & 0,40 & 0,40 & & 0,40 & 0,40 & 0,395 \\
\hline Consistencia Cono & $(\mathrm{mm})$ & 190 & 140 & & 220 & 190 & 270 \\
\hline Diseño Mezcla $R_{b k}$. & $\mathrm{MPa}$ & 30 & 30 & & 30 & 30 & 30 \\
\hline Variante & & $\mathrm{a}$ & $\mathrm{b}$ & $\mathrm{c}$ & $\mathrm{d}$ & $\mathrm{e}$ & $\mathrm{f}$ \\
\hline & $14 \mathrm{~d}$ & 37,5 & 27,6 & 39,04 & 39,04 & 26,2 & 39,3 \\
\hline Resistencia a compresión & $28 \mathrm{~d}$ & 45,9 & 37,6 & 48,1 & 46,2 & 36,9 & 49,90 \\
\hline Rendimiento, 28 días & & 1,14 & 0,94 & 1,2 & 1,15 & $\mathbf{0 , 9 2}$ & 1,24 \\
\hline
\end{tabular}

$\mathrm{N}$ y RC Aditivos superplastificantes para el prefabricado y premezclado respectivamente

Tabla No. 8 Resultados de los Ensayos en hormigones

\begin{tabular}{|c|c|c|c|c|c|c|}
\hline \multirow{2}{*}{ Identificación } & \multicolumn{6}{|c|}{ Valores promedio a 28 días } \\
\cline { 2 - 7 } & $\begin{array}{c}\text { Absorción } \\
\text { de agua } \\
(\mathrm{g})\end{array}$ & $\begin{array}{c}\text { Velocidad } \\
\text { ultrasónica } \\
(\mathrm{m} / \mathrm{s})\end{array}$ & $\begin{array}{c}\text { Masa } \\
\text { Volumétrica } \\
\left(\mathrm{t} . \mathrm{m}^{-3}\right)\end{array}$ & $\begin{array}{c}\mathrm{m} \\
\left(\mathrm{s} / \mathrm{m}^{2}\right)\end{array}$ & $\begin{array}{c}\mathrm{K} \\
\left(\mathrm{kg} . / \mathrm{m}^{2} . \mathrm{s}^{1 / 2}\right)\end{array}$ & $\varepsilon_{\mathrm{e}}(\%)$ \\
\hline $\mathrm{N}$ & 5,3 & 4510 & 2,31 & $7 \times 10^{7}$ & $1.368 \times 10^{-2}$ & 8,36 \\
\hline $\mathrm{G}$ & 4,1 & 4551 & 2,34 & $5 \times 10^{7}$ & $1.588 \times 10^{-2}$ & 9,54 \\
\hline $\mathrm{NP}$ & 6,0 & 4438 & 2,30 & $6 \times 10^{7}$ & $1.614 \times 10^{-2}$ & 10,45 \\
\hline $\mathrm{RC}$ & 12,6 & 4636 & 2,32 & $8 \times 10^{7}$ & $1.472 \times 10^{-2}$ & 11,05 \\
\hline $\mathrm{M}$ & 5,0 & 4398 & 2,30 & $7 \times 10^{7}$ & $1.028 \times 10^{-2}$ & 9,92 \\
\hline $\mathrm{RP}$ & 6,0 & 4510 & 2,31 & $7 \times 10^{7}$ & $1.516 \times 10^{-2}$ & 10,80 \\
\hline
\end{tabular}

\section{DISCUSION}

Con la utilización de los compuestos de nanosílice se obtienen incrementos en la laborabilidad para igual relación $\mathrm{a} / \mathrm{c}$, incrementos notables en la resistencia a compresión y excelente rendimiento sobre todo a tempranas edades (Tabla 7), dado que al tener el compuesto partículas muy finas la reacción se incrementa por el efecto de mayor superficie de contacto.

Los valores de la absorción de agua por capilaridad según la norma JIS A 1404, se encuentran muy por debajo de la especificación, (30 gramos), la porosidad efectiva $\varepsilon_{\mathrm{e}}$ menor del $10 \%$ y la velocidad ultrasónica mayor de $4000 \mathrm{~m} / \mathrm{s}$ denotan un incremento en la compacidad, libre de 
Revista ALCONPAT, Volumen 2, Número 2, Mayo - Agosto 2012, Páginas 82 - 88

oquedades, capilares y/o grietas, así mismo la obtención de las mayores coeficientes de resistencia a la penetración del agua, $\mathrm{m}\left(\mathrm{s} / \mathrm{m}^{2}\right)$ y menores coeficientes de absorción capilar determinan un incremento de la calidad y durabilidad al empleo de la nanosílice respecto a los hormigones tradicionalmente empleados con aditivos y adiciones (Tabla 8).

Por otro lado el empleo de compuesto de nanosílice además se obtienen ventajas para el medio ambiente y para los trabajadores que con estos polvos fino laboran, reduciendo la emisión de éstos a la atmósfera y evitando la inhalación de los mismos, es una contribución a la utilización de tecnologías limpias y un incremento en la calidad y durabilidad de los hormigones en los que se emplean.

\section{AGRADECIMIENTOS}

Al Ing. Rubén Bayarri la firma ULMEN, Industrias Ulmen Europa S.L Zaragoza, España y al Dr. Ing. Juan José J. Howland Albear de la Dirección de Desarrollo Tecnológico, al primero por el suministro de las muestras y al segundo por la recomendación de la ejecución del trabajo.

\section{REFERENCIAS}

Ficha Técnica Gaia Nanosílice (2009), Edición No.2, Identificación No. 1002 Versión 02.7 p Gann, D. (2002), A review of nanotechnology and its potencial applications for construction, SPRU, University of Sussex, Brighton, Reino Unido.

Industrias Ulmen Europa, S.L. Construcción - hormigones 2p. (2009), Ficha Técnica Gaia Ns Compact, Edición No.2 Mayo 2009. Identificación No. 1001 Versión 014 p

Instrucción del Hormigón Estructural (EHE-08) (2008), (R.D. 1247/2008 - BOE de 18 de julio 2008, núm. 203, pp. 35176- en 35178), entrada vigor el 01 de diciembre de 2008 Instrucción del Hormigón Estructural (EHE-08) (R.D. 1247/2008 - BOE de 18 de julio 2008, núm. 203, pp. 35176- en 35178), entrada vigor el 01 de diciembre de 2008

JIS 1404:1997 (1997), Método de ensayo para adiciones impermeables del cemento en hormigones y morteros.

NC 231: 2002 (2002), Determinación, interpretación y aplicación de la velocidad del pulso ultrasónico en el hormigón.

NC 345:2011 (2011), Hormigón endurecido. Determinación de la absorción de agua por capilaridad que utiliza el método propuesto por Goran Fagerlund

Payá J., Borrachero M. V., Monzó J., Soriano L., Martínez- Velandia D., Bueno A. (2008), Estudios sobre cemento Pórland adicionado con nanosílice. X Congreso Nacional de Materiales (Donostia San Sebastián 18-20 Junio 2008. 6p.

Roco M. C., Williams R. S., Alivisatos P. (1999), Nanotechnology Research Directions: IWGN Research Report. Committee on Technology, Interagency Working Group on Nanoscience, Engineering and Technology (IWGN), National Science and Technology Council

Sobolev K., Ferrada Gutiérrez M. (2005b), How nanotechnology can change the concrete world: Part 2, American Ceramic Society Bulletin, 11, pp. 16-19.

Sobolev K., Ferrada-Gutiérrez M. (2005a), How nanotechnology can change the concrete world: Part 1, American Ceramic Society Bulletin, 10, pp. 14-17.

Talero R., Delgado A. (2009), Los cementos, sus adiciones y la durabilidad del hormigón, en Geomateriales, Materiales de construcción y desarrollo sostenible, Edita Sanfeliú, T, Jordán, M.M. y Excmo. Ayuntamiento de Castellón de La Plana, pp. 295-348, Castellón de La Plana, España. 